\title{
NESTING SURFACES OF CONSTANT RATE OF ENERGY DISSIPATION IN CREEP*
}

\author{
BY \\ C. R. CALLADINE, University of Cambridge \\ AND \\ D. C. DRUCKER, Brown University
}

1. Introduction. Previous work by Hoff [1] on bodies undergoing steady state creep is extended. The general creep law chosen reduces to a single power term form with exponent $n$ for uniaxial tension or simple shear. A comparison is made between homogeneous bodies of arbitrary but identical shape and size, each composed of material of different $n$. In general terms, the result is that the convex surfaces of constant (normalized) dissipation rate, plotted in a (reduced) load space, nest inside each other as $n$ increases. This nesting property can be of great help in the practical calculation of close approximations to creep deflections. All surfaces coincide at points which correspond to a design for minimum weight. Solutions with $n=1$ are the complete analog of linear elastic solutions; those with $n$ infinite correspond to perfectly plastic solutions. [1]

2. Stress-strain relation and dissipation rate. A bar under constant uniaxial stress, at a temperature which is elevated for the material of the bar, usually exhibits a steady rate of deformation or creep following an initial or primary stage of decrease from a much higher value of creep rate. The steady strain rate often is given reasonably well over an appreciable range of stress by a single power term in the stress

$$
\epsilon^{\prime}=B_{N} \sigma^{n}
$$

The constant of proportionally $B_{N}$ and the power $n$ vary greatly from material to material and alter with temperature for a given material.

The stress-strain relation (1) may be put in dimensionless form by any arbitrary choice of a convenient reference state of stress $\sigma_{N}$ and of corresponding strain rate $\epsilon_{N}^{\prime}=B_{N} \sigma_{N}^{n}$ for each material separately.

$$
\frac{\epsilon^{\prime}}{\epsilon_{N}^{\prime}}=\left(\frac{\sigma}{\sigma_{N}}\right)^{n}
$$

The rate of dissipation of energy per unit volume $D=\sigma \epsilon^{\prime}$ in non-dimensional form is $D / D_{N}=\left(\sigma / \sigma_{N}\right)\left(\epsilon^{\prime} / \epsilon_{N}\right)$, where

$$
D_{N}=\sigma_{N} \epsilon_{N}^{\prime}=B_{N} \sigma_{N}^{n+1}
$$

A simple generalization of (1) and (2) for strain rate $\epsilon_{i i}^{\prime}$ under any state of stress $\sigma_{i j}$ is

$$
\epsilon_{i j}^{\prime}=\frac{\partial \psi}{\partial \sigma_{i j}}, \quad \psi=\frac{D_{N}}{n+1} \phi^{n+1},
$$

where the dimensionless function $\phi$ is homogeneous of degree one in $\sigma_{i j} / \sigma_{N}$ and has the value unity when $\sigma_{i j}$ is the uniaxial tension $\sigma_{N}$. In fully dimensionless form

$$
\frac{\epsilon_{i j}^{\prime}}{\epsilon_{N}^{\prime}}=\phi^{n} \frac{\partial \phi}{\partial\left(\sigma_{i j} / \sigma_{N}\right)}
$$

${ }^{*}$ Received July 26, 1961. 
and *

$$
\frac{D}{D_{N}}=\frac{\sigma_{i i}}{\sigma_{N}} \cdot \frac{\epsilon_{i i}^{\prime}}{\epsilon_{N}^{\prime}}=\phi^{n+1}
$$

Surfaces of constant $\phi$ in stress space represent constant rate of dissipation. They are convex [2], similar, and concentric. The strain rate is normal to each surface at the appropriate stress point, and normals along any radial line from the origin are parallel. If the ratio of corresponding radii of two surfaces is $\alpha$ then the ratio of each of the components of strain rate at the corresponding points along a radial line from the origin is $\alpha^{n}$. The ratio of the dissipation rates is $\alpha^{n+1}$.

The incomplete generality of (4) as an extension of (1) will be restricted further by a strong assumption of similarity of the materials to be compared. The functional form of $\phi$ will be assumed to be the same for all. Of course, the materials still may differ both in the value of $n$ and in the multiplicative factor corresponding to $B_{N}$ of (1). In fact, the total restriction is not as severe as might be supposed. Good agreement with the actual steady creep of many metals is obtained with a simple isotropic form of this class, $\psi=C_{Q} J_{2}^{a}$ or $\phi^{2}=3 J_{2} / 2 \sigma_{N}^{2}$, in which $C_{Q}$ is a constant, $J_{2}$ is the second invariant of the stress deviation, and $2 q=n+1$. However, anisotropic forms are as permissible as any of the isotropic forms familiar in the theory of plasticity. It is worth noting that a maximum shearing stress criterion may be employed, in spite of the singularities in the surface of constant $\phi$, because (6) still applies.

3. Description of the problem. A customary and often useful approach [1] to the creep of bodies under constant loads is to assume that secondary or steady creep is of overwhelming importance. Primary creep is ignored, as is the adjustment of stresses from an initial elastic or elastic-plastic response to the steady creep state. Fig. 1 is a schematic illustration of the problem which is posed here. Two bodies of identical shape and size are shown, composed of material with the same governing functional form $\phi$. For the one on the left, $n$ is called $m$, with the accompanying notation $B_{M}, \sigma_{M}, \epsilon_{M}^{\prime}, D_{M}$ and $\phi_{M}$. For the one on the right, $n$ is called $r$ with the accompanying notation $B_{R}, \sigma_{R}, \epsilon_{R}^{\prime}, D_{R}$ and $\phi_{R}$. The body on the left is acted upon by a set of forces $P_{i M}$, the body on the right by a proportional set of loads $P_{i R}$;

$$
\frac{P_{i R}}{\sigma_{R}}=\beta \frac{P_{i M}}{\sigma_{M}} .
$$

Avoidance of infinite stress is symbolized by the loading blocks between the forces and the body in both cases. The boundary condition on velocity $u_{i}^{\prime}$ is that $u_{i}^{\prime}=0$ wherever $u_{i}^{\prime}$ is specified.

Suppose further that the average dimensionless rate of dissipation of energy (6) throughout the volume $V$ is unity for each system. For the body $M$

$$
\int_{V} \phi_{M}^{m+1} d V=V
$$

or

For the body $R$

$$
P_{i M} u_{i M}^{\prime}=\int_{V} \sigma_{i j M} \epsilon_{i j M}^{\prime} d V=\sigma_{M} \epsilon_{M}^{\prime} V=D_{M} V
$$

$$
\int_{V} \phi_{R}^{r+1} d V=V
$$

${ }^{*}$ Repeated lower case subscripts in the same term denote summation. 
or

$$
P_{i R} u_{i R}^{\prime}=\int_{V} \sigma_{i j R} \epsilon_{i j R}^{\prime} d V=\sigma_{R} \epsilon_{R}^{\prime} V=D_{R} V .
$$

Subscripts $M$ and $R$ are employed with $\phi$ to emphasize that $\phi_{M}$ is a function (homogeneous of degree one) of $\sigma_{i j M} / \sigma_{M}$ while $\phi_{R}$ is identically the same function of $\sigma_{i j R} / \sigma_{R}$. For $\sigma_{i j M}=\sigma_{i j R}, \phi_{M}=\phi_{R} \sigma_{R} / \sigma_{M}$. If $\sigma_{i j M}=\gamma \sigma_{i j R}$,

$$
\phi_{M}=\gamma \phi_{R} \sigma_{R} / \sigma_{M} \text {. }
$$

The problem is to determine as much as possible about $\beta$ in (7) as a function of the exponents $m$ and $r$ and to show in particular that if $r>m$ then $\beta \leq 1$.

4. Elastic analog and extremum theorems. If strain rate and velocity are replaced by strain and displacement, each of the steady creep problems of Fig. 1 becomes an

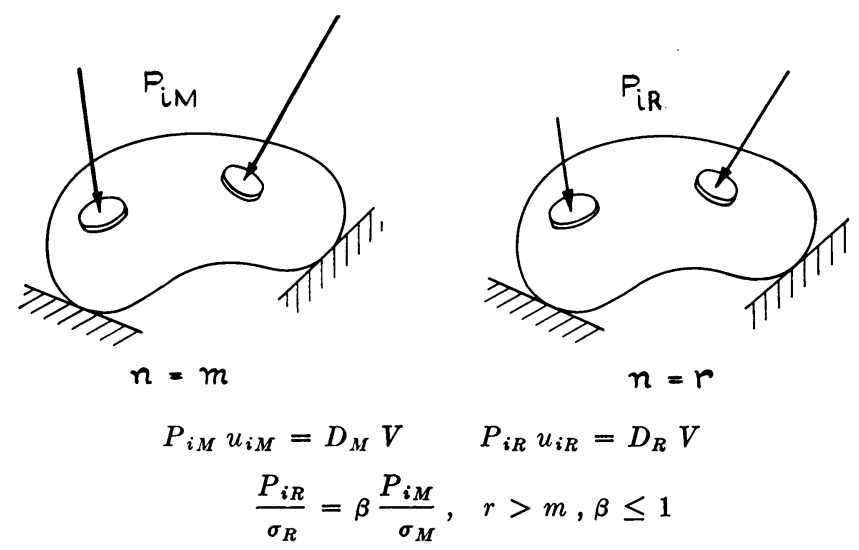

FIG. 1

elastic problem which is linear for $n=1$ and is non-linear in general. The distribution and magnitude of the stresses depend upon the applied loads and the exponent $n$. Strain depends upon $B_{N}$ as well.

If [3] for any given material obeying (5) the loads are multiplied by $\gamma$ the stresses likewise are $\gamma$ times as large while the strains are multiplied by $\gamma^{n}$.

The theorems of minimum potential energy and minimum complementary energy are applicable to the elastic problem and therefore by analogy to the creep problem as well [4]. The equivalent of strain energy density is

$$
W\left(\epsilon_{i j}^{\prime}\right)=\int_{0}^{\epsilon^{\prime} i j} \sigma_{i j} d \epsilon_{i j}^{\prime}=\frac{n}{n+1} \sigma_{i j} \epsilon_{i j}^{\prime}=\frac{n}{n+1} D_{N} \phi_{N}^{n+1}
$$

when (6) is substituted. The equivalent of complementary energy density is

$$
\Omega\left(\sigma_{i j}\right)=\int_{0}^{\sigma_{i j}} \epsilon_{i j}^{\prime} d \sigma_{i j}=\frac{1}{n+1} \sigma_{i j} \epsilon_{i j}^{\prime}=\frac{1}{n+1} D_{N} \phi_{N}^{n+1} .
$$

For the stress boundary condition type of problem described, the theorem of minimum potential energy becomes

$$
\int_{V} W\left(\epsilon_{i j}^{\prime}\right) d V-P_{i} u_{i}^{\prime} \leq \int_{V} W\left(\bar{\epsilon}_{i j}^{\prime}\right) d V-P_{i} \bar{u}_{i}
$$


where $\bar{u}_{i}^{\prime}, \bar{\epsilon}_{i j}^{\prime}$ is any compatible set of velocities and strain rates satisfying the requirement that $\bar{u}_{i}^{\prime}=0$ wherever $u_{i}^{\prime}$ is specified.

The theorem of minimum complementary energy becomes

$$
\int_{\nabla} \Omega\left(\sigma_{i j}\right) d V \leq \int_{V} \Omega\left(\sigma_{i i}^{*}\right) d V
$$

where $\sigma_{i j}^{*}$ is any equilibrium set of stresses satisfying the boundary conditions on stress. As

$$
P_{i} u_{i}^{\prime}=\int_{V} \sigma_{i i} \epsilon_{i j}^{\prime} d V=\int_{V} W\left(\epsilon_{i j}^{\prime}\right) d V+\int_{V} \Omega\left(\sigma_{i j}\right) d V=D_{N} \int_{V} \phi_{N}^{n+1} d V,
$$

after division by $D_{N}(15)$ and (16) can be written as the continued inequality

$$
\frac{n+1}{D_{N}} P_{i} \bar{u}_{i}^{\prime}-n \int_{V} \bar{\phi}_{N}^{n+1} d V \leq \int_{V} \phi_{N}^{n+1} d V \leq \int_{V}\left(\phi_{N}^{*}\right)^{n+1} d V
$$

where $\phi$ is the value of $\phi$ for $\epsilon_{i i}^{\prime}=\bar{\epsilon}_{i j}^{\prime}$ and $\phi^{*}$ is the value of $\phi$ for $\sigma_{i i}=\sigma_{i j}^{*}$ when the exponent in (1) and (5) is $n$.

5. Proof that $\beta \leq 1$ if $\mathrm{r}>\mathrm{m}$. The state of stress for body $R$ is in equilibrium under loads $P_{i R}=\beta P_{i M} \sigma_{R} / \sigma_{M}$. Multiplied by $\gamma=\sigma_{M} / \beta \sigma_{R}$ the stresses form an equilibrium set for body $M$ with loads $P_{i M}$.

The right hand inequality (complementary energy theorem) of (18) is

$$
\int_{V} \phi_{M}^{m+1} d V \leq \int_{V}\left(\phi_{M}^{*}\right)^{m+1} d V
$$

in which the left hand integral (8) equals $V$ and $\phi_{M}^{*}$ from (12) is $\left(\sigma_{M} / \beta \sigma_{R}\right) \phi_{R} \sigma_{R} / \sigma_{M}=\phi_{R} / \beta$. Therefore

$$
V \beta^{m+1} \leq \int_{V} \phi_{R}^{m+1} d V
$$

or

$$
\beta^{m+1} \leq \frac{1}{V} \int_{V}\left(\phi_{R}^{r+1}\right)^{(m+1) /(r+1)} d V
$$

The right hand side of (21) is of the form

$$
\int x^{s} d V
$$

where $x \geq 0$ everywhere. As (22) is monotonically increasing in $s$, and from (10) is equal to unity when $s=1$, when $s<1$ (22) is less than unity. Therefore from (21) if $r>m$, $\beta \leq 1$.

6. Interpretation in (reduced) load space. The meaning of the result for $\beta$ is illustrated in Fig. 2. Surfaces of unit average dimensionless rate of dissipation of energy, $P_{i} u_{i}^{\prime}=D_{N} V$, drawn in a space whose co-ordinates are $P_{i} / \sigma_{N}$ nest inside each other as $n$ increases. When, for a particular $P_{i}, \phi_{R}$ in (20) or (21) is unity everywhere in the body, $\beta=1$ and all surfaces coincide. Considering the perfectly plastic analog, $r \rightarrow \infty$, this necessary and sufficient condition for $\beta=1$ may be recognized as the criterion for minimum weight design: viz. $\sigma_{i j} \epsilon_{i j}^{\prime}$ constant throughout the volume of perfectly plastic material [5]. Similarly, $\phi=1$ everywhere is the criterion for the most effective use of 


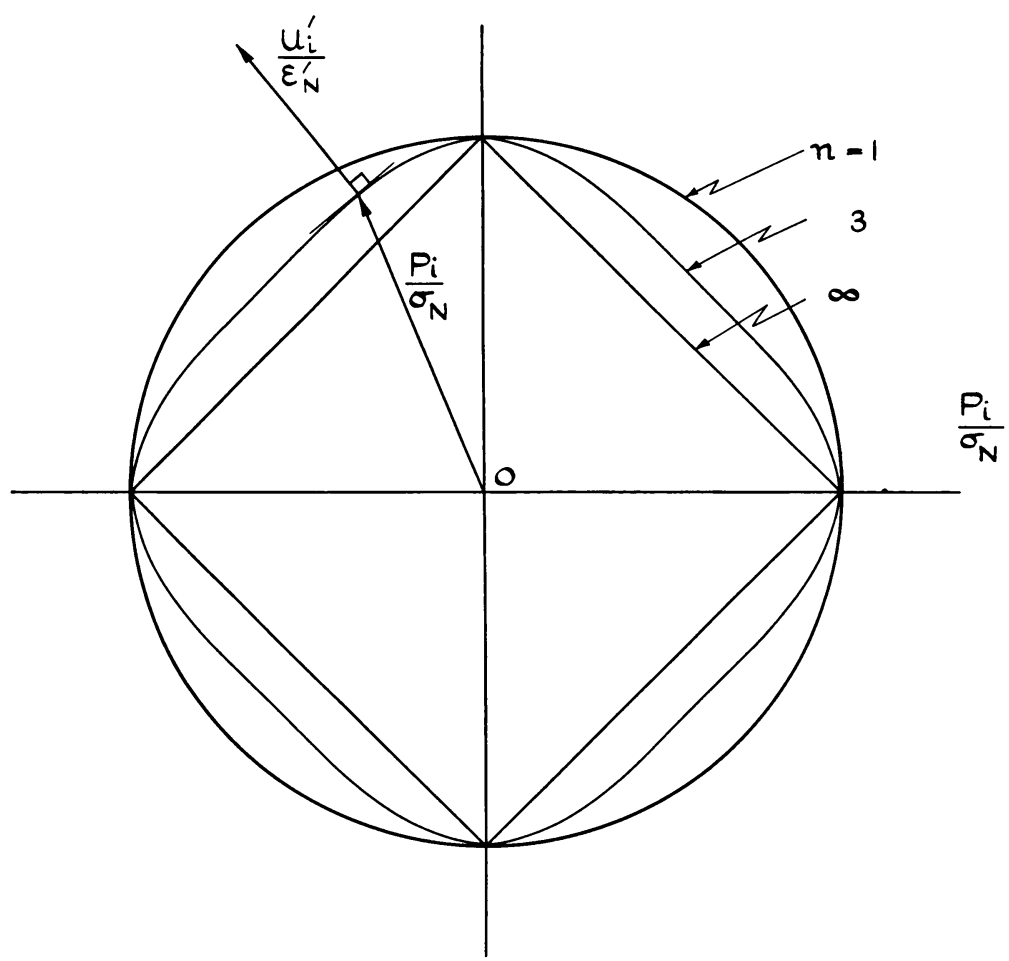

Frg. 2. Nesting surfaces of dissipation rate

$$
P_{i} u_{i}^{\prime}=D_{N} V=\sigma_{N} \epsilon_{N}^{\prime} V \text { or } \frac{P_{i}}{\sigma_{N}} \frac{u_{i}^{\prime}}{\epsilon_{N}}=V \text {. }
$$

material (5) in creep. Just as surfaces of constant $\phi$ are convex in stress space and strain rates are normal to them, the surfaces of constant dissipation rate are convex in $P_{i}$ or $P_{i} / \sigma_{N}$ space and the corresponding working components of velocity are normal to them; Fig. 2. The curves shown in Fig. 2. correspond to the very simple statically determinate two-dimensional structure of Fig. 3. This example is taken from a companion

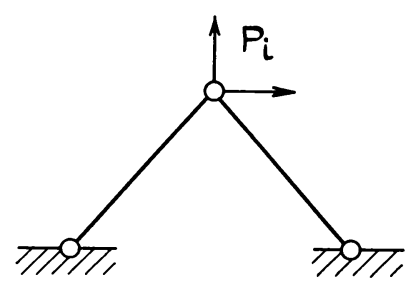

FIg. 3. Structure giving the curves of Fig. 2

paper [6] devoted to the practical use of these properties in problems of analysis of a number of types of structure.

7. Lower and upper bounds on $\beta$. A lower bound on $\beta$ can be found with the theorem of minimum potential energy, the left hand inequality of (18), with $M, m$ for $N, n$

$$
\frac{m+1}{D_{M}} P_{i M} \bar{u}_{i}^{\prime}-m \int_{V} \bar{\phi}_{M}^{m+1} d V \leq \int_{V} \phi^{m+1} d V
$$


It would be permissible to substitute the strain rate $\epsilon_{i j R}^{\prime}$ and velocity $u_{i R}^{\prime}$ for body $R$ as a compatible state for body $M$. It is better, however to multiply these quantities by $\beta \epsilon_{M}^{\prime} / \epsilon_{R}^{\prime}=\left(\beta \sigma_{R} / \sigma_{M}\right)\left(D_{M} / D_{R}\right)$ so that $P_{i M}^{\prime} \bar{u}_{i}^{\prime}=\left(P_{i R} u_{i R}^{\prime}\right)\left(D_{M} / D_{R}\right)=D_{M} V$. Also from (5) $. \bar{\phi}_{M}^{m}=\phi_{R}^{r}$ for $\bar{\epsilon}_{i j M}^{\prime} / \epsilon_{M}^{\prime}=\epsilon_{i j R}^{\prime} / \epsilon_{R}^{\prime}$ or $\bar{\epsilon}_{i j M}^{\prime}=\left(\epsilon_{i i R}^{\prime}\right)\left(\epsilon_{M}^{\prime} / \epsilon_{R}^{\prime}\right)$. For strain rates $\beta$ times as large $\bar{\phi}_{M}=\beta^{1 / m} \phi_{R}^{r / m}$. Thus (23) can be written

$$
(m+1) V-m \beta^{(m+1) / m} \int_{V} \phi_{R}^{(m+1) r / m} d V \leq V
$$

or

$$
\frac{V}{\int_{V} \phi_{R}^{(m+1) r / m} d V} \leq \beta^{(m+1) / m}
$$

A more symmetric form in $r$ and $m$ is obtained if the exponent $(m+1) r / m$ is written as $(r+1)(r / m)[(m+1) /(r+1)]$. For $r>m$ the exponent is greater than $r+1$ so that, as it should be, the left hand side of (25) is less than unity (see (22)).

The complementary forms to (21) and (25) are convenient at times. They can be written down by inspection as

$$
\frac{V}{\int_{V}\left(\phi_{M}^{m+1}\right)^{(r+1) /(m+1)} d V} \leq \beta^{r+1}
$$

and

$$
\beta^{(r+1) / r} \leq \frac{1}{V} \int_{V}\left(\phi_{M}^{m+1}\right)^{(m / r)(r+1) /(m+1)} d V
$$

8. Generalized stresses and strains. All the results carry through without essential change if the stress-strain relation is replaced by a generalized stress-generalized strain relation with the same exponent $n$. For example, axial force in a bar or bending moment in a beam may be substituted for $\sigma$ in (1) or (2) and $\epsilon^{\prime}$ replaced by rate of elongation or rate of change of curvature respectively. Equivalent substitutions are made for sheets, plates and shells.

9. Velocity boundary condition problem. The convex surfaces of constant unit average dissipation rate in $u_{i}^{\prime} / \epsilon_{N}^{\prime}$ space follow an inverse order from those in $P_{i} / \sigma_{N}$ space. They nest inside each other as $n$ decreases.

10. Acknowledgement. This paper was written while one of the authors (D.C.D.) was on sabbatic leave from Brown University. Grateful acknowledgement is made to Brown University for this leave and to the John Simon Guggenheim Memorial Foundation for a fellowship.

\section{REFERENCES}

1. N. J. Hoff, "Approximate analysis of structures in the presence of moderately large creep deformations" Q. Appl. Math. 12, 49-55 (1954)

2. D. C. Drucker, A definition of stable inelastic material, J. Appl. Mech. 26, 101-106 (1959)

3. W. Prager, Total creep under varying loads, J. Aero. Sci. 24, 153-155 (1957); a generalization of a theorem by Ilyushin.

4. S. A. Patel, B. Venkatraman and P. G. Hodge, Jr., Torsion of cylindrical and prismatic bars in the presence of steady creep, J. Appl. Mech. 25, 214-218 (1958)

5. D. C. Drucker and R. T. Shield, Bounds on minimum weight design Q. Appl. Math. 15, 269-281 (1957)

6. C. R. Calladine and D. C. Drucker, $A$ bound method for creep analysis of structures: Direct use of solutions in elasticity and plasticity, to appear in the Journal of Mech. Engrg. Sci. 